\title{
A Decade of Expanding Rural Health and Human Services to the Underserved: The National Consortium of Telehealth Resource Centers
} Tory Cenaj

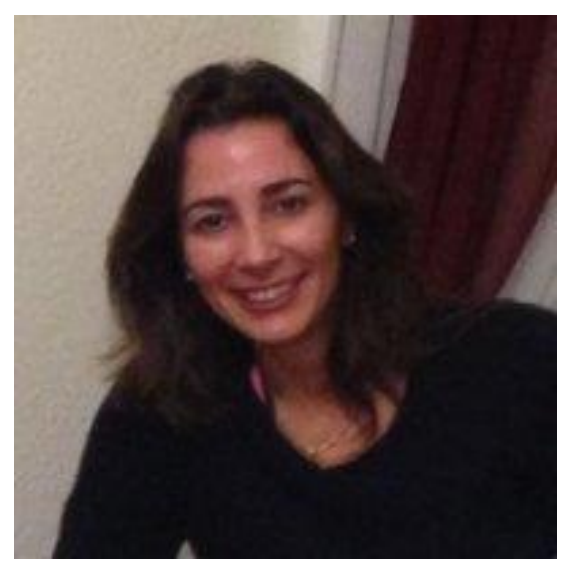

In its policy brief of March 2015, the National Advisory Committee on Rural Health and Human Services ${ }^{1}$ recognized that, "For progress to be made and monitored, regulatory changes must be made to include more telehealth services and reimbursements for those services." Importantly, the Committee also acknowledged that "quality must be reported and monitored."

For a decade, the National Consortium of Telehealth Resource Centers has advanced telehealth toward the goals of telemedicine program implementation and telemedicine process improvement by providing the nation with comprehensive, unbiased information and education related to telehealth. Each Telehealth Resource Centers (TRC) is a nonprofit organization whose charge is to facilitate expansion of telehealth and the availability of healthcare through telemedicine for rural and underserved populations.

\section{National Consortium of Telehealth Resource Centers}

The TRCs emerged out of the general successes experienced by the original Office for the Advancement of Telehealth-supported telehealth network grant programs. Today, there are fourteen TRCs, including twelve Regional Centers whose combined coverage reaches all 50 states, the District of Columbia, and the affiliated pacific islands. In 
addition, two National TRCs focus on technology assessment and telehealth policy, offering their services to the entire United States (Figure 1). TRCs work collaboratively and participate in a consortium known as the National Consortium of TRCs to provide information and assistance to all requesters.

The uniqueness of each TRC allows them to provide assistance targeted to their regional needs and constituencies, while their collaborative efforts provide a national, consistent voice on national telehealth issues. As more health systems and providers look to telehealth to support their patients, the TRCs become even more valuable for their knowledgeable, unbiased voice.

Contributions to telehealth by TRCs have focused on technical assistance in legal/regulatory, business model, evaluation, best practices, technology assessment, equipment, and program development.

\section{The Future}

The TRCs continue to evolve along with healthcare in America. Over the next 3 to 5 years, technologies for mHealth, Population Based Health, and Remote Patient Monitoring will dominate the telehealth and healthcare industry. As these technologies gain greater visibility and popularity, the TRCs stand ready to provide an experienced, reliable voice to help all participants in the health system make informed decisions.

Contact the Center for Connected Health Policy at 877.707.7172 for more information. The National Telehealth Technology Assessment Resource Center can be contacted at 877.885.5672. A sampling of services provided by each regional TRC and links to their websites are presented in Table 1. 


\section{Table 1. Telehealth Resource Centers: Areas \& Services Provided?}

\section{CTRC: California Telehealth Resource Center}

California

- Website with tools, templates, and information to establish telehealth services

- Telehealth Program Developer

- $\quad$ Specialty provider listing, with 69 licensed California telehealth service providers

\section{gpTRAC: Great Plains Telehealth Resource \&}

\section{Assistance Center}

North and South Dakota, Minnesota, lowa, Wisconsin, Nebraska

- Regional collaborator and knowledge source

- Highlight regional telehealth efforts

- Connect regional telehealth leaders to raise awareness of and collaborate on telehealth issues of mutual interest/value

- Disseminate telehealth-related policy and regulatory information

- $\quad$ Provide information, guidance, and assistance to support organizations developing telehealth services

\section{HTRC: Heartland Telehealth Resource Center}

Kansas, Missouri, Oklahoma

- $\quad$ eSTART Assessment for on-site comprehensive analysis of ability to sustain a telehealth program

- Nurses outreach visa \#NurseTechMonday

MATRC: Mid-Atlantic Telehealth Resource Center

Virginia, West Virginia, Kentucky, Maryland, Delaware, North Carolina, Pennsylvania, Washington DC, New Jersey (shared)

- Technical assistance/consultation

- $\quad$ State-specific and topical resources covering programs, policies and services

- Inventory of telehealth providers

- $\quad$ State strategic planning support to address priority health needs through telehealth

- Annual Regional Telehealth Summit

\section{NETRC: Northeast Telehealth Resource Center}

Connecticut, Maine, Massachusetts, New Hampshire, New Jersey (shared), New York, Rhode Island, Vermont

- Interactive website to assist and inform telehealth efforts throughout the region and beyond

- Comprehensive Resource Library with over 1,200 searchable resources 
- Telehealth Basics Curriculum for training on telehealth and telepresenting

- $\quad$ State pages featuring Telehealth Reimbursement Guides specific to the policy/regulatory environment in each state

\section{NRTRC: Northwest Regional Telehealth Resource Center}

Washington, Oregon, Idaho, Montana, Utah, Wyoming, Alaska

- " $\quad$ Open Mic" webinars and the NRTRC Blog, focused on key components of successful programs

- $\quad$ NRTRC toolkits for program development, including:

- Telemental Health Toolkit, a collaboration with UMTRC

- Telehealth Marketing Toolkit

- $\quad$ NRTRC whitepapers assist customers plan and implement programs

\section{PBTRC: Pacific Basin Telehealth Resource Center}

Hawaii, Pacific Basin

- $\quad$ Tele-genetics training courses with the Hawaii Department of Health, SWTRC and SCTRC

- Integration of direct secure messaging into Pacific Islands Emergency Medical Services for Children processes and procedures for pediatric emergency medical evacuations

- Support providers for Rural Health Care Program and Healthcare Connect Fund

- Identify malpractice and reimbursement policies

- $\quad$ Track policy changes

\section{SCTRC: South Central Telehealth Resource Center}

Arkansas, Mississippi, Tennessee

- $\quad$ Over 85 videos available

- Patient vignettes, featured site stories and explainer series

- "Jennifer's Story: A Telemedicine Tale" has had over 30,000 views

\section{SETRC: Southeastern Telehealth Resource Center}

Alabama, Georgia, South Carolina and Florida

- $\quad$ State Telehealth Workgroups address barriers, increase telehealth awareness, and promote state-wide collaboration

- $\quad$ SETRC's virtual workforce training center, the National School of Applied TeleHealth (NSAT) - delivers on-line, standardized, accredited, and affordable telehealth instruction

- Telemedicine/Telehealth courses instruct on essentials of telehealth and prepare individuals to become valuable members of a telehealth team

\section{SWTRC: Southwestern Telehealth Resource Center}

Arizona, Colorado, New Mexico, Nevada, Utah 
- Two full-day courses:

- "Developing a Telemedicine Program" surveys telemedicine topics including clinical services, telecommunications and infrastructure, development/ operations, evaluation, business aspects, distance education, and equipment demonstration

- "Telemedicine Applications" a more in-depth look at clinical applications

\title{
$\underline{\text { TLTRC: }}$ TexLa Telehealth Resource Center
}

\author{
Texas, Louisiana \\ Telehealth Certificate and Simulated Learning Program includes online \\ modules, simulation center learning, and objective structured clinical \\ examinations (OSCEs) to create a competency-based model of \\ education

\section{UMTRC Upper Midwest Telehealth Resource Center} \\ Indiana, Illinois, Michigan, Ohio \\ Archived webinars on its YouTube channel \\ Hosts the National TRC webinar series and posts those archived \\ webinars on the National TRC YouTube channel
}

Ms. Cenaj is Founder and Publisher of Telehealth and Medicine Today ${ }^{\mathrm{TM}}$, and Principal,

Partners in Digital Health.

Tags: California Telehealth Resource Center, Center for Connected Health Policy, Great Plains Telehealth Resource \& Assistance Center, Heartland Telehealth Resource Center, how does telehealth enhance the doctor patient relationship, National Advisory Committee on Rural Health and Human Services, National Consortium of Telehealth Resource Centers, Northeast Telehealth Resource Center, Northwest Regional Telehealth Resource Center, Pacific Basin Telehealth Resource Center, South Central Telehealth Resource Center, Southeastern Telehealth Resource Center, Southwestern Telehealth Resource Center, telehealth, telemedicine, telemedicine process improvement, telemedicine program implementation, telemedicine remote health, TexLa Telehealth Resource Center, TRC, Upper Midwest Telehealth Resource Center, what is telemedicine and how does it work, National Telehealth Technology Assessment Resource Center 


\section{References}

1. National Advisory Committee on Rural Health and Human Services. Telehealth in Rural America. Policy Brief. March 2015. URL:

http://www.hrsa.gov/advisorycommittees/rural/publications/telehealthmarch2015. pdf. Accessed 7/26/16.

2. The National Consortium of Telehealth Resource Centers. A decade of service. 2016. URL:https://www.nrtrc.org/content/articlefiles/White\%20Papers/TRC\%2010\%20Year\%20Report\%20digital.pdf. Accessed $8 / 22 / 16$ 


\section{TelehealthResourceCenters.org}

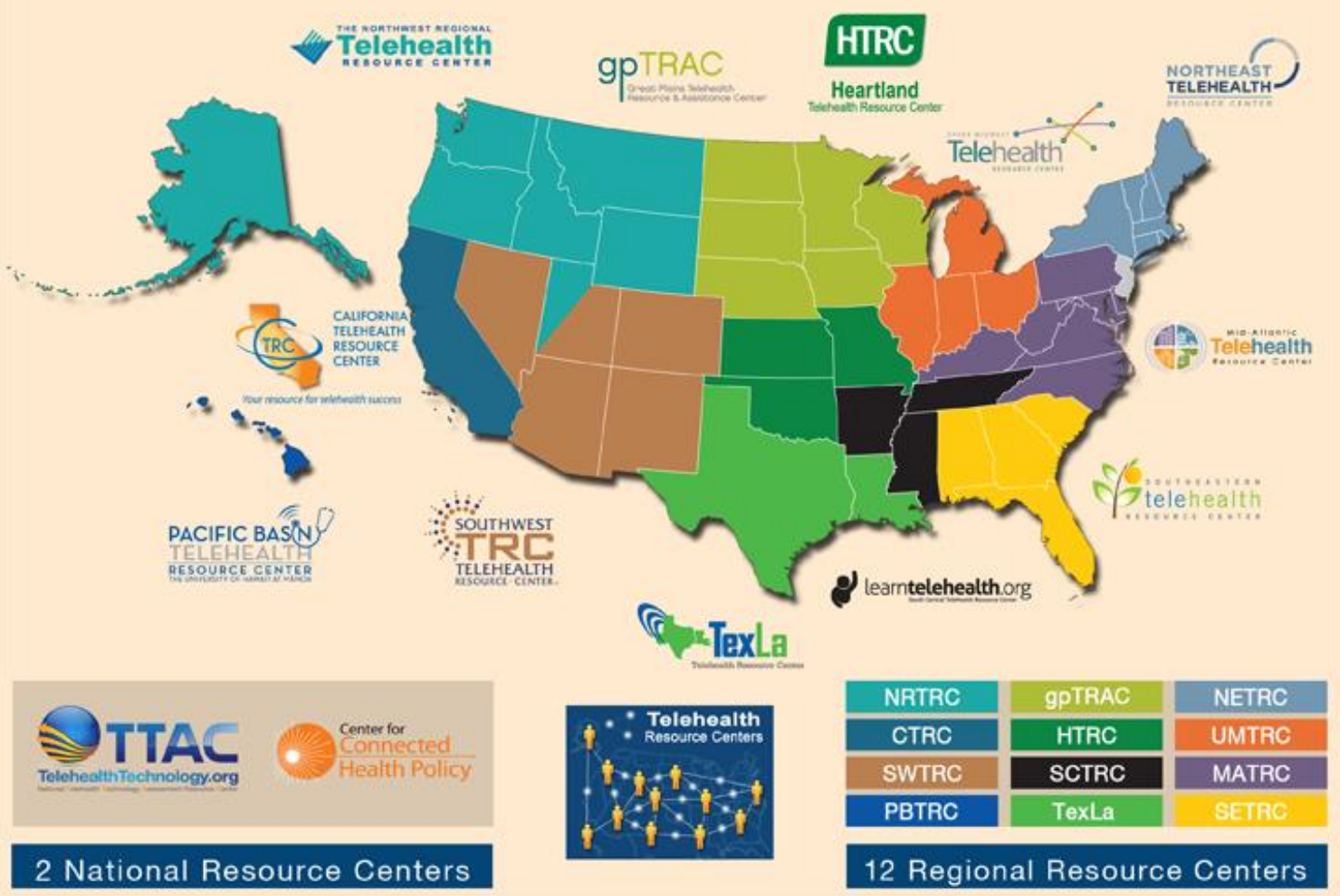

Figure 1. Telehealth Resource Centers include 2 national and 12 regional centers. Used with permission of the National Telehealth Technology Assessment Resource Center. URL: http://www.telehealthtechnology.org/resources. 


\section{Table 1. Telehealth Resource Centers}

\section{CTRC: California Telehealth Resource Center}

California

- Website to help establish telehealth services

- Telehealth Program Developer

- Specialty provider listing

\section{gpTRAC: Great Plain Telehealth Resource \& Assistance} Center

North and South Dakota, Minnesota, lowa, Wisconsin, Nebraska

- Regional collaborator and knowledge source

- Disseminate telehealth-related policy and regulatory information

- Provide assistance for developing telehealth services

\section{HTRC: Heartland Telehealth Resource Center}

Kansas, Missouri, Oklahoma

- On-site analysis of ability to sustain a telehealth program

- Nurses outreach visa \#NurseTechMonday

MATRC: Mid-Atlantic Telehealth Resource Center

Virginia, West Virginia, Kentucky, Maryland, Delaware, North Carolina, Pennsylvania, Washington DC, New Jersey (shared)

- Technical assistance/consultation

- State-specific and topical resources

- Annual Regional Telehealth Summit

\section{NETRC: Northeast Telehealth Resource Center}

Connecticut, Maine, Massachusetts, New Hampshire, New Jersey (shared), New York, Rhode Island, Vermont

- Resource Library with over 1,200 searchable resources

- Telehealth Curriculum to train on telehealth and telepresenting

- Telehealth Reimbursement Guides

\section{NRTRC: Northwest Regional Telehealth Resource}

\section{Center}

Washington, Oregon, Idaho, Montana, Utah, Wyoming, Alaska

- "Open Mic" webinars and the NRTRC Blog

- Toolkits for program development

- $\quad$ NRTRC whitepapers to plan and implement programs 


\section{Table 2. Telehealth Resource Centers (cont'd)}

\section{PBTRC: Pacific Basin Telehealth Resource Center}

Hawaii, Pacific Basin

- Tele-genetics training

- Integrate secure messaging into Pacific Islands Emergency Medical Services for pediatric evacuations

- Track policy changes

\section{SCTRC: South Central Telehealth Resource Center}

Arkansas, Mississippi, Tennessee

- Over 85 videos available

- Patient vignettes, featured site stories and explainer series

- "Jennifer's Story: A Telemedicine Tale" with over 30,000 views

SETRC: Southeastern Telehealth Resource Center

Alabama, Georgia, South Carolina and Florida

- State Telehealth Workgroups

- SETRC's virtual workforce training center

- Telemedicine/Telehealth courses on essentials of telehealth

SWTRC: Southwestern Telehealth Resource Center

Arizona, Colorado, New Mexico, Nevada, Utah

- Two full-day courses:

- "Developing a Telemedicine Program"

- "Telemedicine Applications"

\section{TLTRC: TexLa Telehealth Resource Center}

Texas, Louisiana

- Telehealth Certificate and Simulated Learning Program

\section{UMTRC Upper Midwest Telehealth Resource Center}

Indiana, Illinois, Michigan, Ohio

- Archived webinars on its YouTube channel

- Hosts the National TRC webinar series and posts those archived webinars on the National TRC YouTube channel 